ITP-Budapest Report 597

\title{
SUSY sine-Gordon theory as a perturbed conformal field theory and finite size effects
}

\author{
Z. Bajnok ${ }^{1}$, C. Dunning ${ }^{2}$, L. Palla ${ }^{3}$, G. Takács ${ }^{1}$ and F. Wágner ${ }^{3,4}$ \\ ${ }^{1}$ Theoretical Physics Research Group, Hungarian Academy of Sciences, Budapest, Hungary \\ ${ }^{2}$ Department of Mathematics, University of York, York, United Kingdom \\ ${ }^{3}$ Institute for Theoretical Physics, Eötvös University, Budapest, Hungary \\ ${ }^{4}$ Department of Mathematics, King's College London, London, United Kingdom
}

1st February 2008

\begin{abstract}
We consider SUSY sine-Gordon theory in the framework of perturbed conformal field theory. Using an argument from Zamolodchikov, we obtain the vacuum structure and the kink adjacency diagram of the theory, which is cross-checked against the exact $\mathrm{S}$ matrix prediction, first-order perturbed conformal field theory (PCFT), the NLIE method and truncated conformal space approach. We provide evidence for consistency between the usual Lagrangian description and PCFT on the one hand, and between PCFT, NLIE and a massgap formula conjectured by Baseilhac and Fateev, on the other. In addition, we extend the NLIE description to all the vacua of the theory.
\end{abstract}

\section{Introduction}

Supersymmetric (SUSY) sine-Gordon theory (SSG) is of interest for several reasons. First, it is a two-dimensional integrable field theory and as such, there is a wealth of analytic and non-perturbative information available about its behaviour. Second, it describes supersymmetric solitons, which have been investigated recently in the context of supersymmetric gauge theories in four (and other) dimensions. It can be expected that SSG provides a useful laboratory for the analysis of solitons in supersymmetric theories, and their nonperturbative behaviour in general. Third, there has been a lot of activity recently especially concerning the situation when SSG has a nontrivial boundary condition imposed on a timelike boundary. 
In spite of all the progress, much less is known about SSG than its non-supersymmetric counterpart, i.e. ordinary sine-Gordon theory. Although its S matrix has been conjectured several years ago [1], there is much less evidence for its correctness than in the non-SUSY

case. In particular, the issue of quantum corrections to the soliton mass has been settled only fairly recently in [2], where it was proven that it satisfies a BPS-like property, even though the theory only has $N=1$ supersymmetry.

There is also a certain controversy in the literature concerning the vacuum and kink structure of the tricritical Ising model [3], the simplest nontrivial SUSY integrable field theory, which has a bearing on the S matrix of the SSG as well, since the S matrix of tricritical Ising model provides the description of the supersymmetric structure.

In this paper we set out to clarify the vacuum and kink structure of the model (without boundaries), using information from several sources. First, in Section 3 we apply an argument going back to Zamolodchikov [4] which gives a kink structure compatible with the S matrix conjectured in [1]. In Section 4 SSG is considered in the perturbed conformal field theory framework, which is not entirely trivial, as a purely bosonic potential term has to be omitted from the Lagrangian (it is expected to be generated by radiative corrections in this description). To show that this description is correct we construct a spin-3 conserved charge and verify it against the classical limit. In addition, we analyze first-order perturbative corrections of the lowest-lying energy levels, and in Section 5 compare them to the results obtained from a conjectured NLIE, first derived in [5], which is extended to provide a finite volume description of all the vacuum levels. Finally, in Section 6 we use the truncated conformal space method to investigate the finite volume spectrum. Our conclusions are presented in Section 7.

\section{SSG theory}

\subsection{Action and discrete symmetries}

The supersymmetric sine-Gordon (SSG) theory is defined by the action

$$
\mathcal{A}_{\mathrm{SSG}}=\int d t d x\left(\frac{1}{2} \partial_{\mu} \Phi \partial^{\mu} \Phi+i \bar{\Psi} \gamma^{\mu} \partial_{\mu} \Psi+m \bar{\Psi} \Psi \cos \frac{\beta}{2} \Phi+\frac{m^{2}}{\beta^{2}} \cos \beta \Phi\right)
$$

where $\Phi$ is a real scalar, $\Psi$ is a Majorana fermion field, $m$ is a mass parameter and $\beta$ is the coupling constant. The theory is invariant under an $N=1$ supersymmetry algebra and has infinitely many commuting local conserved charges [6]. These charges survive at the quantum level and render the theory integrable, which makes it possible to describe the exact spectrum and the $\mathrm{S}$ matrix. We use the Weyl representation for the spinor field

$$
\begin{aligned}
\gamma^{0} & =\left(\begin{array}{cc}
0 & i \\
-i & 0
\end{array}\right) \quad, \quad \gamma^{1}=\left(\begin{array}{cc}
0 & i \\
i & 0
\end{array}\right) \quad, \quad \gamma^{3}=\gamma^{0} \gamma^{1}=\left(\begin{array}{cc}
-1 & 0 \\
0 & 1
\end{array}\right) \\
\Psi & =\left(\begin{array}{l}
\psi_{-} \\
\psi_{+}
\end{array}\right)
\end{aligned}
$$


where $\psi_{ \pm}$are real Weyl components with definite chirality.

The supersymmetric theory has some discrete symmetries that play an important role in what follows. The field theory interaction is periodic

$$
\Phi \rightarrow \Phi+n \frac{4 \pi}{\beta}, \quad n \in \mathbb{Z}
$$

and even in the boson field

$$
\Phi \rightarrow-\Phi
$$

These symmetries are also present in the non-supersymmetric version of the sine-Gordon model. It is also interesting to observe that the Lagrangian is invariant under a half-period shift

$$
\Phi \rightarrow \Phi+\frac{2 \pi}{\beta}
$$

if at the same time one changes the relative sign of the fermion components, say

$$
\psi_{+} \rightarrow-\psi_{+} \quad, \quad \psi_{-} \rightarrow \psi_{-}
$$

or

$$
\Psi \rightarrow-\gamma^{3} \Psi
$$

\subsection{Spectrum and scattering amplitudes}

The spectrum consists of the soliton/antisoliton multiplet, realizing supersymmetry in a nonlocal way, and breathers that are bound states of a soliton with an antisoliton.

The building blocks of supersymmetric factorized scattering theory were first described in [7], using an Ansatz in which the full scattering amplitude is a direct product of a part carrying the SUSY structures and a part describing all the rest of the dynamics. The full SSG S matrix was constructed in [1].

The supersymmetric solitons are described by RSOS kinks $K_{a b}^{\epsilon}(\theta)$ of mass $M$ and rapidity $\theta$, where $a, b$ take the values $0, \frac{1}{2}$ and 1 with $|a-b|=1 / 2$, and describe the supersymmetric structure, while $\epsilon= \pm$ corresponds to topological charge \pm 1 (soliton/antisoliton). Multi-particle asymptotic states are built as follows

$$
\left|K_{a_{0} a_{1}}^{\epsilon_{1}}\left(\theta_{1}\right) K_{a_{1} a_{2}}^{\epsilon_{2}}\left(\theta_{2}\right) \ldots K_{a_{N-2} a_{N-1}}^{\epsilon_{N-1}}\left(\theta_{N-1}\right) K_{a_{N-1} a_{N}}^{\epsilon_{N}}\left(\theta_{N}\right)\right\rangle
$$

where $\theta_{1}>\theta_{2}>\ldots>\theta_{N}$ for an in state and $\theta_{1}<\theta_{2}<\ldots<\theta_{N}$ for an out state. The two-particle scattering process

$$
K_{a b}^{\epsilon_{1}}\left(\theta_{1}\right)+K_{b c}^{\epsilon_{2}}\left(\theta_{2}\right) \rightarrow K_{a d}^{\epsilon_{2}^{\prime}}\left(\theta_{2}\right)+K_{d c}^{\epsilon_{1}^{\prime}}\left(\theta_{1}\right)
$$

has an amplitude of the form

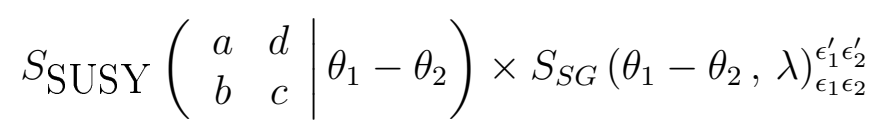


i.e. the tensor structure of the scattering amplitude factorizes into a part describing the SUSY structure (which we call the SUSY factor) and another part corresponding to the topological charge (the bosonic factor).

The bosonic factor coincides with the usual sine-Gordon $\mathrm{S}$ matrix, but the relation between the parameter $\lambda$ and $\beta$ is different from the sine-Gordon case

$$
\lambda=\frac{8 \pi}{\beta^{2}}-\frac{1}{2} .
$$

The SUSY factor is identical to the S matrix of the tricritical Ising model perturbed by the primary field of dimension $\frac{6}{5}[4]$ :

$$
\begin{aligned}
& S_{\mathrm{SUSY}}\left(\begin{array}{cc|c}
0 & \frac{1}{2} & \theta \\
\frac{1}{2} & 0 & \theta
\end{array}\right)=S_{\mathrm{SUSY}}\left(\begin{array}{cc|c}
1 & \frac{1}{2} & \theta \\
\frac{1}{2} & 1 & \theta
\end{array}\right)=2^{(i \pi-\theta) / 2 \pi i} \cos \left(\frac{\theta}{4 i}-\frac{\pi}{4}\right) K(\theta) \\
& S_{\mathrm{SUSY}}\left(\begin{array}{cc|c}
\frac{1}{2} & 0 & \theta \\
0 & \frac{1}{2} & \theta
\end{array}\right)=S_{\mathrm{SUSY}}\left(\begin{array}{cc|c}
\frac{1}{2} & 1 & \theta \\
1 & \frac{1}{2} & \theta
\end{array}\right)=2^{\theta / 2 \pi i} \cos \left(\frac{\theta}{4 i}\right) K(\theta) \\
& S_{\mathrm{SUSY}}\left(\begin{array}{cc|c}
0 & \frac{1}{2} & \theta \\
\frac{1}{2} & 1 & \theta
\end{array}\right)=S_{\mathrm{SUSY}}\left(\begin{array}{cc|c}
1 & \frac{1}{2} & \theta \\
\frac{1}{2} & 0 & \theta
\end{array}\right)=2^{(i \pi-\theta) / 2 \pi i} \cos \left(\frac{\theta}{4 i}+\frac{\pi}{4}\right) K(\theta) \\
& S_{\mathrm{SUSY}}\left(\begin{array}{cc|c}
\frac{1}{2} & 1 & \theta \\
0 & \frac{1}{2} & \theta
\end{array}\right)=S_{\mathrm{SUSY}}\left(\begin{array}{cc|c}
\frac{1}{2} & 0 & \theta \\
1 & \frac{1}{2} & \theta
\end{array}\right)=2^{\theta / 2 \pi i} \cos \left(\frac{\theta}{4 i}-\frac{\pi}{2}\right) K(\theta) \\
& K(\theta)=\frac{1}{\sqrt{\pi}} \prod_{k=1}^{\infty} \frac{\Gamma\left(k-\frac{1}{2}+\frac{\theta}{2 \pi i}\right) \Gamma\left(k-\frac{\theta}{2 \pi i}\right)}{\Gamma\left(k+\frac{1}{2}-\frac{\theta}{2 \pi i}\right) \Gamma\left(k+\frac{\theta}{2 \pi i}\right)} .
\end{aligned}
$$

The supersymmetry factor has no poles in the physical strip and so the full supersymmetric amplitudes of the kinks have the same poles as in the ordinary sine-Gordon model. The corresponding bound states are supersymmetric breathers $B_{n}$ of mass

$$
m_{n}=2 M \sin \frac{\pi n}{2 \lambda} \quad, \quad n=1, \ldots,[\lambda]
$$

which transform in the ordinary boson-fermion doublet representation of the SUSY algebra. The bosonic component of $B_{n}$ has a nontrivial $(-1)^{n}$ parity under bosonic field reflection $\Phi \rightarrow-\Phi$.

\section{Kink and vacuum structure of SUSY models}

Here we use an argument by Zamolodchikov [4] to clarify the vacuum and kink structure of SUSY sine-Gordon theory. In its original form the argument is for the tricritical Ising model; we briefly recall it here. 


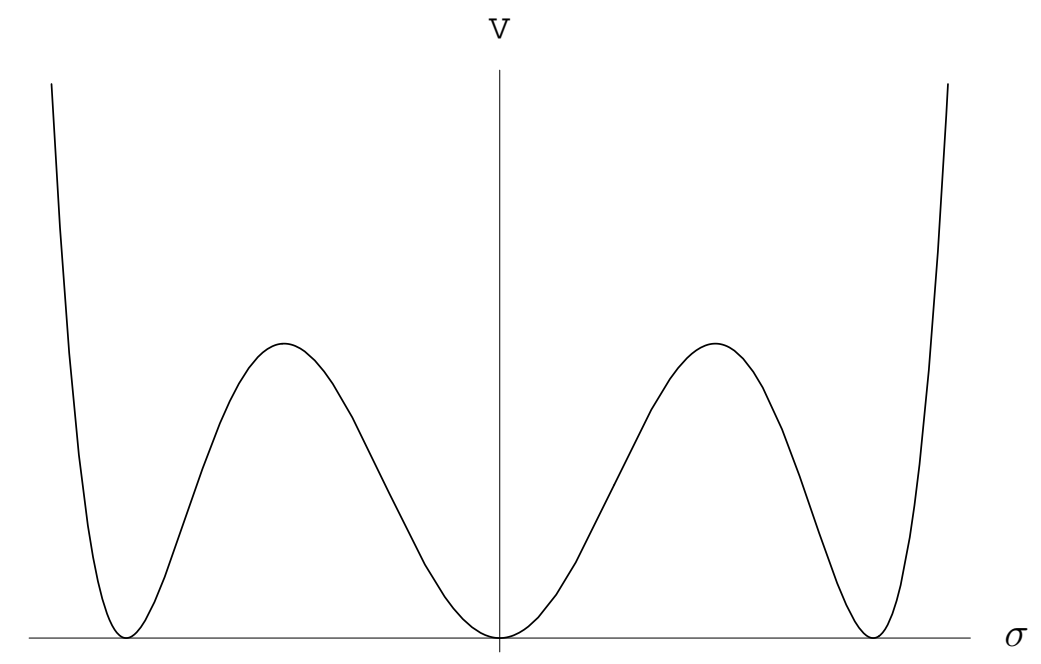

Figure 3.1: The scalar potential in the Landau-Ginzburg description of the tricritical Ising model

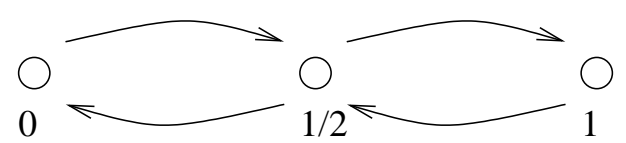

Figure 3.2: The adjacency diagram of kinks in the tricritical Ising model

\subsection{Tricritical Ising model}

In the bosonic Landau-Ginzburg formulation, the basic field is the (bosonic) spin field $\sigma$, and the action is of the form

$$
\int\left(\frac{1}{2}\left(\partial_{t} \sigma\right)^{2}-\frac{1}{2}\left(\partial_{x} \sigma\right)^{2}-V(\sigma)\right) d x d t
$$

where the potential has the form

$$
V(\sigma)=\lambda\left(\sigma^{2}-\sigma_{0}^{2}\right)^{2} \sigma^{2} .
$$

As can be seen from the form of the potential (Fig. 3.1), there are three vacua in the model, and the kinks connect them according to the adjacency diagram in Fig. 3.2, with a scattering amplitude given in (2.8).

However, in the supersymmetric Landau-Ginzburg formalism the action (in component form) looks like

$$
\int\left(\frac{1}{2}\left(\partial_{t} \Phi\right)^{2}-\frac{1}{2}\left(\partial_{x} \Phi\right)^{2}+\psi_{+} \partial_{-} \psi_{+}+\psi_{-} \partial_{+} \psi_{-}+W^{\prime}(\Phi) \psi_{+} \psi_{-}+W(\Phi)^{2}\right) d x d t
$$




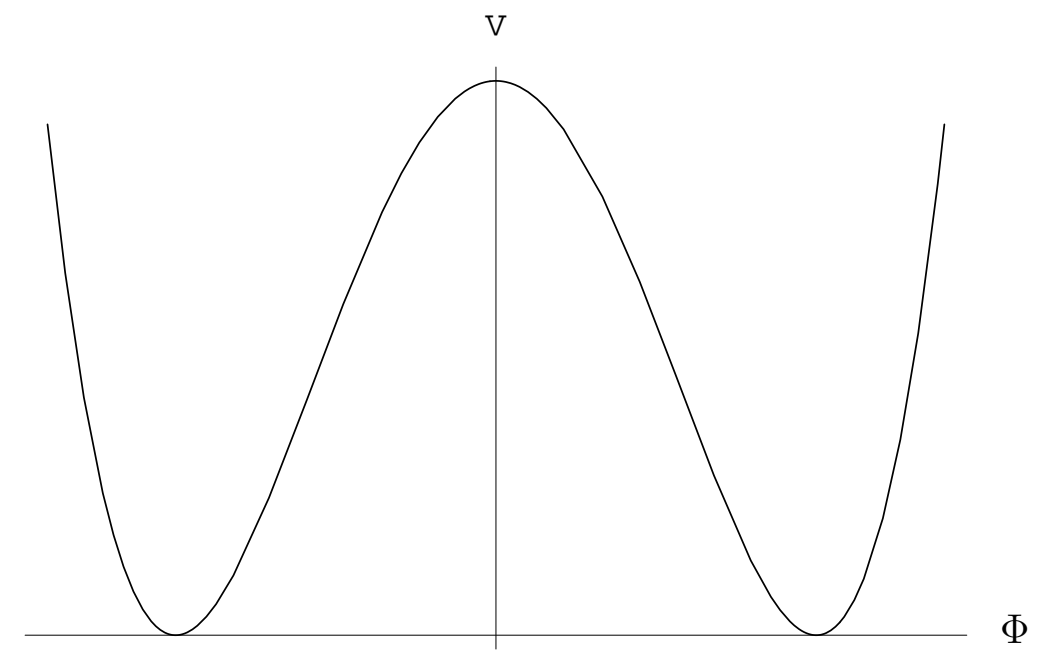

Figure 3.3: The scalar potential in the SUSY LG description of the tricritical Ising model

where

$$
W(\Phi)=\mu\left(\Phi^{2}-\lambda^{2}\right) .
$$

The scalar potential is of the form (Fig 3.3 )

$$
V(\Phi)=\mu^{2}\left(\Phi^{2}-\lambda^{2}\right)^{2}
$$

and has two minima

$$
\Phi= \pm \lambda .
$$

How do we reconcile this picture with the bosonic one, in which there are three vacua? Zamolodchikov's argument goes as follows: at the minimum $\Phi=\lambda$, the fermion mass is positive, there is only one vacuum, the Majorana fermion describes the high temperature phase of the Ising model and the vacuum expectation value of the spin field vanishes:

$$
\langle\sigma\rangle=0 .
$$

However, when $\Phi=-\lambda$, we are in the low temperature phase of the Ising model. Therefore this vacuum is twofold degenerate, with

$$
\langle\sigma\rangle= \pm \sigma_{0} .
$$

The kinks go from the $\langle\sigma\rangle=0$ vacuum to the $\langle\sigma\rangle= \pm \sigma_{0}$ ones. Therefore there are two pairs of kinks, and we get the same adjacency diagram (Fig. 3.2), with the $\langle\sigma\rangle=0$ (RSOS label $\frac{1}{2}$ ) vacuum in the middle, while the other two $\langle\sigma\rangle= \pm \sigma_{0}$ (RSOS labels 0,1$)$ sit at the edges.

It must be mentioned that Fendley has conjectured another bootstrap S matrix for the tricritical Ising model in [3], based on an assumption that the kink structure in the SUSY 
Landau-Ginzburg formulation was different from that of the bosonic formulation. The bosonic and the super Landau-Ginzburg formulations are two different local descriptions of the model; their relation is very similar to the sine-Gordon/massive Thirring connection [8]. However, in the case of the sine-Gordon/massive Thirring relation the kink S matrix is independent of the local description taken, and Zamolodchikov's argument indicates that this is the case for the tricritical Ising model as well. As a result, in both the bosonic and the fermionic description the kink scattering is described by the same RSOS S matrix (2.8), contrary to Fendley's assumption. ${ }^{1}$ The difference between the local algebras manifests itself in the different boundary conditions prescribed for the local fields, just as in the sine-Gordon/massive Thirring case (cf. also [9] for the situation in finite volume).

\section{2 $\mathrm{N}=1$ SUSY sine-Gordon theory}

Now let us apply these considerations to $N=1$ SUSY sine-Gordon theory. Here the bosonic potential is

$$
-\cos \beta \Phi
$$

while the mass term of the fermion is of the form

$$
\psi_{-} \psi_{+} \cos \frac{\beta \Phi}{2} .
$$

As a result, every even vacuum $\Phi=2 n \frac{2 \pi}{\beta}, n \in Z$ is nondegenerate, while every odd vacuum $\Phi=(2 n+1) \frac{2 \pi}{\beta}, n \in Z$ is doubly degenerate, with nonzero expectation value of the spin field associated to the Majorana fermion. We have a vacuum structure analogous to that of tricritical Ising model, which periodically repeats itself (similarly to the periodic vacuum structure of the sine-Gordon model). This results in the adjacency diagram shown in Fig. 3.4, which is exactly the one that describes the tensor product $\mathrm{S}$ matrix of kinks $K_{a b}^{ \pm}, a=0, \frac{1}{2}, 1$.

The discrete symmetries of the Lagrangian are manifest on the adjacency diagram, which is periodic in $\Phi$ with period $4 \pi / \beta$. The transformation $(2.4,2.5)$ is a translation with $2 \pi / \beta$, together with the analogue of Kramers-Wannier duality acting on the Majorana fermion, and exchanges the broken phase of the fermion (two vacua) and the symmetric phase (a single vacuum).

Due to the periodicity of the vacuum structure and the field theoretic potential, in finite volume one can create twisted sectors depending on a $\vartheta$ angle according to [10], or one can define folded versions of the model by identifying vacua after a certain number of periods [11].

\footnotetext{
${ }^{1}$ TBA results also show that Fendley's S matrix yields an irrational value for the ultraviolet central charge, thus it cannot correspond to a perturbation of tricritical Ising model (M. Moriconi, private communication).
} 


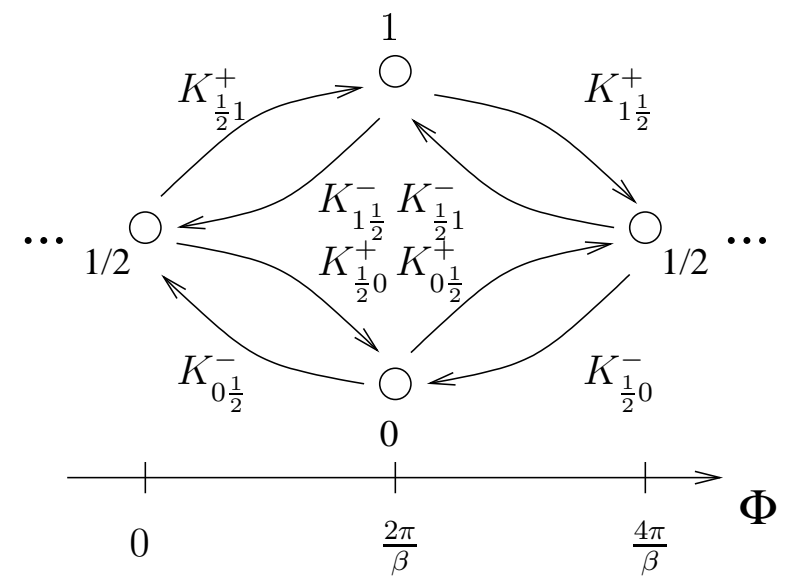

Figure 3.4: A period of the adjacency diagram of kinks in supersymmetric sine-Gordon theory

\subsection{The IR description}

Now we give a qualitative description of the consequences of imposing periodic boundary conditions in the SSG theory in the IR domain, i.e in a large but finite volume $L$. It is based on the known features of the spectrum and the exact $\mathrm{S}$ matrix.

The first consequence of imposing periodic boundary conditions is that the single kink states are excluded. Some of the two kink states are allowed; namely those $K_{a b} K_{b c}$ where the $a$ and $c$ vacua are identical. As two kinks can fuse into breathers one may have single breather states in the sectors where the topological charge vanishes. It is easy to see, using the $a \rightarrow 1-a$ symmetry of the SUSY factor (2.8), that the allowed two kink states are divided into two sets,

$$
\left(K_{0 \frac{1}{2}} K_{\frac{1}{2} 0}, K_{1 \frac{1}{2}} K_{\frac{1}{2} 1}\right) \quad\left(K_{\frac{1}{2} 0} K_{0 \frac{1}{2}}, K_{\frac{1}{2} 1} K_{1 \frac{1}{2}}\right)
$$

closed under scattering. The first set contains two vacua (the ones indexed by 0 and 1 ), while the second one has a single vacuum (the one indexed by $\frac{1}{2}$ ). It is a natural expectation that in a finite volume these three vacua are no longer degenerate but their energies are exponentially close to each other. Above all three vacua we also expect the repetition of the spectrum of (single) breather states albeit with their energies split. One of the aims of this paper is to give a qualitative verification of this picture.

The two sets of periodic two kink states have rather different supersymmetry properties. To establish this we use the kink-kink-breather fusion coefficients

$$
\left|K_{a b}(\theta+i \xi / 2) K_{b c}(\theta-i \xi / 2)\right\rangle=f_{a b c}^{\phi}|\phi(\theta)\rangle+f_{a b c}^{\psi}|\psi(\theta)\rangle,
$$

(where $|\phi\rangle$ and $|\psi\rangle$ stand for the bosonic and fermionic breathers respectively) explicitly given in [12]. These explicit expressions reveal that the two kink states in the first set can fuse into bosonic breathers only, while the ones in the second set can fuse into both 
bosonic and fermionic breathers. Furthermore, using the action of supersymmetry charges (also given in [12]), one can show that there is an unbroken $N=1$ supersymmetry in the space of states of the second set.

\section{SSG as a perturbed conformal field theory}

\subsection{The perturbed conformal field theory picture}

We consider SUSY sine-Gordon theory as a perturbation of the $c=3 / 2$ conformal field theory of a free boson and a free fermion. The Hilbert space of the theory is taken to be a tensor product of the conformal free boson and free fermion, and we consider only local sectors, i.e. retain only states which have integer conformal spin. At generic values of the compactification radius this permits only two boundary conditions on the fermion field

$$
\Psi(x+L)= \pm \Psi(x)
$$

which means either Neveu-Schwarz or Ramond boundary conditions on both components of the fermion fields (different boundary conditions on the components would result in sectors of noninteger spin).

We take a conformal boson field $\phi$ compactified on a circle of radius $r$, normalized with the action

$$
\frac{1}{8 \pi} \int d^{2} z \partial \phi \bar{\partial} \phi
$$

which means that the canonical boson field of (2.1) can be written as $\Phi=\frac{1}{\sqrt{4 \pi}} \phi$. The conformal boson has a $\hat{U}(1) \times \hat{U}(1)$ symmetry generated by the modes of the currents

$$
J(z)=i \partial \phi=\sum_{n} a_{n} z^{-n-1} \quad \text { and } \quad \bar{J}(\bar{z})=i \bar{\partial} \phi=\sum_{n} \bar{a}_{n} \bar{z}^{-n-1}
$$

The highest weight vectors $|n, m\rangle$ of the current algebra are created by the vertex operators

$$
V_{(n, m)}(z, \bar{z})=: e^{i\left(\frac{n}{r}+\frac{m r}{2}\right) \varphi(z)+i\left(\frac{n}{r}-\frac{m r}{2}\right) \bar{\varphi}(\bar{z})}: \text {. }
$$

In order to have a maximal local operator algebra we take both $n$ and $m$ to be integers. The Hamiltonian, on the cylinder of circumference $L$, in the sector $|n, m\rangle$ has the following form

$$
H_{\text {boson }}=\frac{2 \pi}{L}\left(\left(\frac{n}{r}\right)^{2}+\left(\frac{m r}{2}\right)^{2}+\sum_{k>0}\left(a_{-k} a_{k}+\bar{a}_{-k} \bar{a}_{k}\right)-\frac{1}{12}\right),
$$

where the bosonic modes satisfy

$$
\left[a_{k}, a_{l}\right]=k \delta_{k+l} \quad ; \quad\left[a_{k}, \bar{a}_{l}\right]=0 \quad ; \quad\left[\bar{a}_{k}, \bar{a}_{l}\right]=k \delta_{k+l} .
$$


In the Neveu-Schwarz sector, the fermionic part of the Hilbert space can be generated by the negative modes of the Euclidean fermionic fields (corresponding to the Minkowski fields $\psi_{ \pm}$)

$$
\psi(z)=\sum_{s+1 / 2 \in Z} b_{s} z^{-s-1 / 2} ; \bar{\psi}(\bar{z})=\sum_{s+1 / 2 \in Z} \bar{b}_{s} \bar{z}^{-s-1 / 2}
$$

acting on the Neveu-Schwarz vacuum $|N S\rangle$ and locality requires the total fermion number to be even. The modes satisfy

$$
\left\{b_{s}, b_{t}\right\}=\delta_{s+t} \quad ; \quad\left\{b_{s}, \bar{b}_{t}\right\}=0 \quad ; \quad\left\{\bar{b}_{s}, \bar{b}_{t}\right\}=\delta_{s+t} .
$$

The fermionic Hamiltonian takes the form (using conformal normalization conventions)

$$
H_{N S}=\frac{1}{2 \pi} \int d^{2} z \psi \bar{\partial} \psi=\frac{2 \pi}{L}\left(\sum_{s=\frac{1}{2}}^{\infty} b_{-s} b_{s}-\frac{1}{24}\right) .
$$

In the Ramond sector, the fermionic field has integer mode expansion and both the left and the right fermion number must be even. We denote the highest weight state by $|R\rangle$. The Hamiltonian in the Ramond sector is

$$
H_{R}=\frac{2 \pi}{L}\left(\sum_{s=1}^{\infty} b_{-s} b_{s}+\frac{1}{48}\right)
$$

Writing $\cos \frac{\beta}{2} \Phi=\frac{1}{2}\left(V_{(1,0)}+V_{(-1,0)}\right)$ gives the relation $\frac{1}{r}=\frac{\beta}{4 \sqrt{\pi}}$ between the compactification radius $r$ and the coupling $\beta$, and this leads to

$$
p \equiv \frac{1}{\lambda}=\frac{2}{r^{2}-1} .
$$

\subsection{Conserved charges in the PCFT framework and the perturb- ing potential}

A convenient way to describe the SUSY sine-Gordon model is to consider it as an appropriate perturbation of the conformal field theory in its UV limit [13] by the operator $U_{1}=\bar{\psi} \psi \cos \frac{\beta \Phi}{2}$. In [14] the SSG is described as a perturbation of the super Liouville theory and it is shown that the purely bosonic piece of the potential $U_{2}=\frac{m^{2}}{\beta^{2}} \cos \beta \Phi$ vanishes in the renormalized theory.

The problem is that assuming the kinetic terms of the boson and fermion fields in the Lagrangian provide the description of the $c=\frac{3}{2}$ theory, the two parts of the interaction $U_{1}=\bar{\psi} \psi \cos \frac{\beta \Phi}{2}$ and $U_{2}$ have different conformal dimensions: $\Delta_{1}=\bar{\Delta}_{1}=\frac{1}{2}+\frac{\beta^{2}}{32 \pi}$ and $\Delta_{2}=\bar{\Delta}_{2}=\frac{\beta^{2}}{8 \pi}$. This, and the fact that they have different supersymmetry properties would complicate the PCFT description. Nevertheless - using the conserved quantities we argue below that for the SSG it is enough to consider only $U_{1}$ as a perturbation. 
This perturbation obviously preserves a $(1,1)$ supersymmetry generated by the supercurrents $G$ and $\bar{G}$, since in the superfield formalism $U_{1}$ can be written as $G_{-1 / 2} \bar{G}_{-1 / 2} \cos \left(\frac{\beta}{2} \hat{\Phi}\right)$ with $\cos \left(\frac{\beta}{2} \hat{\Phi}\right)$ being a Neveu-Schwarz superconformal primary field [15]. Thus the real question is whether $U_{1}$ provides an integrable perturbation of the $c=\frac{3}{2}$ theory. For this it is enough if $U_{1}$ preserves a single higher spin conserved quantity.

One can look for a conserved spin 3 quantity generated by the density $T_{4}$, where

$$
T_{4}=\left(\partial_{z}^{2} \Phi\right)^{2}+A\left(\partial_{z} \Phi\right)^{4}+B\left(\partial_{z} \Phi\right)^{2} \partial_{z} \psi \psi+C \partial_{z}^{2} \psi \partial_{z} \psi,
$$

with constants $A, B$ and $C$. In the first order of the perturbation $T_{4}$ is conserved if in the operator product $T_{4}(z) U_{1}(w, \bar{w})$ the residue of the first order pole is a total derivative [13]. Having computed the residue one can eliminate the terms containing the derivatives of the $\psi$ field by using total derivatives; the remaining (non total derivative) terms then have the form

$$
\left(\tilde{A} \psi \partial_{z}^{3} \Phi+\tilde{B} \psi \partial_{z}^{2} \Phi \partial_{z} \Phi+\tilde{C} \psi\left(\partial_{z} \Phi\right)^{3}\right) \cos \left(\frac{\beta}{2} \Phi\right) .
$$

Demanding the vanishing of $\tilde{A}, \tilde{B}$ and $\tilde{C}$ yields a linear inhomogeneous system of equations for $A, B, C$, that has a solution, which shows that $U_{1}$ generates an integrable perturbation. Furthermore the $\beta \rightarrow 0$ limits of $A, B, C$ reproduce the classical expressions obtained in [16] using both $U_{1}$ and $U_{2}$ as perturbations together with the classical equations of motion. This provides the justification for only using $U_{1}$ as the perturbation in the PCFT framework.

Therefore in terms of the canonically normalized fields the Lagrangian of the SSG is written in the perturbed CFT framework as

$$
\mathcal{L}=\frac{1}{2} \partial_{\mu} \Phi \partial^{\mu} \Phi+i \bar{\Psi} \gamma^{\mu} \partial_{\mu} \Psi+\mu \bar{\Psi} \Psi \cos \frac{\beta}{2} \Phi .
$$

The coefficient of the perturbing potential is denoted here by $\mu$ to emphasize that its ( $\beta$ dependent) dimension is different from that of the classical mass $m$. Identifying the bulk SUSY sine-Gordon model with the $n=2$ case of [17] gives the following relation between the kink mass $M$ and the $\mu$ parameter

$$
\frac{\mu}{8} \gamma\left(\frac{1}{2}-\frac{\beta^{2}}{32 \pi}\right)=M^{1-\frac{\beta^{2}}{16 \pi}}\left(\frac{\pi}{4} \frac{\beta^{2}}{16 \pi-\beta^{2}}\right)^{1-\frac{\beta^{2}}{16 \pi}}, \quad \gamma(x)=\frac{\Gamma(x)}{\Gamma(1-x)} .
$$

This relation - which connects an IR and a UV parameters and is called the massgap relation - plays an important role in writing the TCSA program devised to analyze the finite volume spectrum of SSG. Note that for $\beta \rightarrow 0$, the massgap relation correctly reproduces the classical kink (soliton) mass $M=\frac{8 \mu}{\beta^{2}}$.

\subsection{The PCFT analysis of energy levels in the UV}

The first PCFT correction to the UV energy levels can be expressed in terms of certain integrals over the complex plane of various $2 \mathrm{p}$ and $4 \mathrm{p}$ functions in the underlying conformal 
field theory [18]. Indeed writing the (bare) Euclidean action of the perturbed CFT on the cylinder as

$$
\mathcal{A}_{g}=\mathcal{A}_{\mathrm{CFT}}+g \int d^{2} \xi \chi(\xi)
$$

where $g$ has mass dimension $y=2-2 \Delta_{\chi}$ and $\chi(z)$ is normalized by $\langle\chi(z, \bar{z}) \chi(0,0)\rangle=$ $|z|^{-4 \Delta_{\chi}}$, for small dimensionless volumes $l=L M$, the first correction to the energy of a state $|a\rangle$, with $L_{0}|a\rangle=\Delta_{a}|a\rangle=\bar{L}_{0}|a\rangle$, can be written as

$$
\frac{6 l}{\pi} E_{a}(l)=-\left(c-24 \Delta_{a}\right)-c_{2}^{a} \kappa^{2} l^{2 y}+o\left(l^{4 y}\right) .
$$

Here $c$ is the central charge of the UV CFT, it is assumed that the symmetry properties of the fields forbid any first order $\left(o\left(l^{y}\right)\right)$ corrections and to derive this formula the massgap relation is written in the form $g=\kappa M^{y}$. We remark that the bulk energy constant in SSG vanishes on account of the supersymmetry. The coefficient of the leading correction at $l \rightarrow 0$ can be written explicitly as

$$
c_{2}^{a}=6(2 \pi)^{1-2 y} \int_{\text {plane }} \frac{d^{2} z}{|z|^{y}}\langle a|\chi(1,1) \chi(z, \bar{z})| a\rangle \mid \text { conn . }
$$

Using the conformal normalized free fermions $\psi, \bar{\psi}$ and the properly normalized $V_{( \pm 1,0)}$ vertex operators to describe the perturbation in the SUSY sine-Gordon model one obtains

$$
\chi(z, \bar{z})=\bar{\psi}(\bar{z}) \psi(z) \frac{1}{\sqrt{2}}\left(V_{(1,0)}(z, \bar{z})+V_{(-1,0)}(z, \bar{z})\right)
$$

and

$$
g=\frac{\mu}{2 \pi \sqrt{2}} .
$$

This perturbation is relevant if the compactification radius $r>1$, and then $|0\rangle,|1 \pm\rangle,|2 \pm\rangle$ and $|3 \pm\rangle$ constitute most of the lowest lying states in the various NS sectors. (Here $|N \pm\rangle$, $N=1,2,3$ denote the states $|N \pm\rangle=\frac{1}{\sqrt{2}}\left(V_{(N, 0)}(0,0)|0\rangle \pm V_{(-N, 0)}(0,0)|0\rangle\right)$ with $|0\rangle$ being the tensor product of the bosonic and NS vacua $|0\rangle=|\tilde{0}\rangle \otimes|N S\rangle)$. For these states it is straightforward to determine the conformal correlation functions needed in eq.(4.2), and the integrals can be evaluated using the general expression

$$
\int d^{2} z \frac{|z|^{\rho-2}}{|1-z|^{2 \nu}}=\pi \gamma\left(\nu-\frac{\rho}{2}\right) \gamma\left(\frac{\rho}{2}\right) \gamma(1-\nu) .
$$

(The integral is convergent at $z \rightarrow 0$ if $\rho>0$, at $z \rightarrow 1$ if $\nu<1$ and at $z \rightarrow \infty$ if $2 \nu-\rho>0$; we keep the conditions $2 \nu>\rho>0$ but continue in $\nu$ above $\nu=1$ using the r.h.s. if necessary). The coefficients of the leading PCFT corrections for these seven states are summarized in Table 4.1, where the coefficient $\alpha$ is

$$
\alpha=\frac{3}{2}(8)^{\frac{2}{r^{2}}} \frac{1}{\gamma^{2}\left(\frac{1}{2}-\frac{1}{2 r^{2}}\right)}\left(\frac{1}{r^{2}-1}\right)^{2-\frac{2}{r^{2}}} .
$$




\begin{tabular}{|c|c|c|}
\hline State & $\Delta_{a}$ & $c_{2}^{a} \kappa^{2}$ \\
\hline$|0\rangle$ & 0 & $\alpha \gamma^{2}\left(\frac{1}{2}+\frac{1}{2 r^{2}}\right) \gamma\left(-\frac{1}{r^{2}}\right)$ \\
\hline$|1 \pm\rangle$ & $\frac{1}{2 r^{2}}$ & $\alpha \gamma\left(\frac{1}{2}-\frac{1}{2 r^{2}}\right)\left[\gamma\left(-\frac{1}{r^{2}}\right) \gamma\left(\frac{1}{2}+\frac{3}{2 r^{2}}\right) \pm \frac{1}{2} \gamma\left(\frac{1}{2}-\frac{1}{2 r^{2}}\right) \gamma\left(\frac{1}{r^{2}}\right)\right]$ \\
\hline$|2 \pm\rangle$ & $\frac{2}{r^{2}}$ & $\alpha \gamma\left(\frac{1}{2}-\frac{3}{2 r^{2}}\right) \gamma\left(-\frac{1}{r^{2}}\right) \gamma\left(\frac{1}{2}+\frac{5}{2 r^{2}}\right)$ \\
\hline$|3 \pm\rangle$ & $\frac{9}{2 r^{2}}$ & $\alpha \gamma\left(\frac{1}{2}-\frac{5}{2 r^{2}}\right) \gamma\left(-\frac{1}{r^{2}}\right) \gamma\left(\frac{1}{2}+\frac{7}{2 r^{2}}\right)$ \\
\hline
\end{tabular}

Table 4.1: The first PCFT corrections for some states

Note that in all these cases it is necessary to make the analytical continuation in $\nu$ to give meaning to the otherwise divergent integrals. This divergence is expected as the conformal dimension of the perturbing operator $\Delta=\frac{1}{2}+\frac{1}{2 r^{2}}$ is greater than $1 / 2$; thus the singularity coming at $z \rightarrow 1$ from the $\chi(1) \chi(z)$ OPE is not integrable no matter what the states $|a\rangle$ are.

In the Ramond sector we expect that the ground state scaling function vanishes identically as a result of unbroken supersymmetry. The question is whether the (leading) PCFT corrections are consistent with this expectation. The conformal contribution to the scaling function vanishes since

$$
c-24 \Delta_{R}=\frac{3}{2}-24 \frac{1}{16}=0 .
$$

The next contribution is proportional to the second order coefficient

$$
c_{2}^{|R\rangle}=6(2 \pi)^{1-2 y} \int_{\text {plane }} \frac{d^{2} z}{|z|^{y}}\langle R|\chi(1,1) \chi(z, \bar{z})| R\rangle \mid \text { conn }
$$

where

$$
|R\rangle=\lim _{z, \bar{z} \rightarrow 0} \sigma(z, \bar{z})|N S\rangle
$$

denotes the Ramond ground state. The integrand defining $c_{2}^{|R\rangle}$ is formally positive, but the integral is divergent and needs regularization. Separating the integrand into a sum of terms, after an appropriate partial integration it can be converted into a form in which each term can be regularized using (4.4). The sum of regularized terms turns out to vanish. In this sense PCFT is consistent with a vanishing ground state scaling function in the Ramond sector.

\section{$5 \quad$ NLIE}

To probe the behaviour of the scaling functions between the ultraviolet and infrared limits one can use the thermodynamic Bethe Ansatz [19,20]. Fendley and Intriligator's TBA gluing idea [21] leads to a set of TBA equations for all choices of the SSG coupling [22-24], but in practice the equations take a simple form only at certain $\beta$. Instead one can treat all 
values of the coupling on an equal footing by using an alternative type of nonlinear integral equation, originally developed for the ground state of the sine-Gordon model in [25] (and independently in a related context in [26,27]), and which usually goes by the acronym NLIE.

The equations proposed in [5] for the SSG model consist of a TBA-like function coupled to a nonlinear integral equation. The two pieces reflect the factorized nature of the S matrix, with the TBA part associated to the SUSY factor and the NLIE part to the bosonic factor. The original equations of [5] generate the lowest ground state in the Neveu-Schwarz sector, but a simple sign change and appropriate choice of parameters provides access to the other two ground states. The equations are

$$
\begin{aligned}
\ln y_{1}(\theta)= & -i l \sinh (\theta)+i \pi \omega+\int_{-\infty}^{\infty} d \theta^{\prime} \chi\left(\theta-\theta^{\prime}+\frac{i \pi}{2}\right) \ln \left(1+(-1)^{\delta} y_{2}\left(\theta^{\prime}\right)\right) \\
& +\int_{\mathcal{C}_{1}} d \theta^{\prime} \varphi\left(\theta-\theta^{\prime}\right) \ln \left(1+y_{1}\left(\theta^{\prime}\right)\right)-\int_{\mathcal{C}_{2}} d \theta^{\prime} \varphi\left(\theta-\theta^{\prime}\right) \ln \left(1+y_{1}^{-1}\left(\theta^{\prime}\right)\right) \\
\ln y_{2}(\theta)= & \int_{\mathcal{C}_{1}} d \theta^{\prime} \chi\left(\theta-\theta^{\prime}-\frac{i \pi}{2}\right) \ln \left(1+y_{1}\left(\theta^{\prime}\right)\right)-\int_{\mathcal{C}_{2}} d \theta^{\prime} \chi\left(\theta-\theta^{\prime}-\frac{i \pi}{2}\right) \ln \left(1+y_{1}^{-1}\left(\theta^{\prime}\right)\right) .
\end{aligned}
$$

The integrations contours $\mathcal{C}_{1}$ and $\mathcal{C}_{2}$ run from $-\infty$ to $\infty$ just below and above the real axis respectively. The kernel $\varphi(\theta)$ is proportional to the logarithmic derivative of the soliton-soliton scattering amplitude of the sine-Gordon model

$$
\varphi(\theta)=\int \frac{d k}{2 \pi} e^{i k \theta} \frac{\sinh (p-1) \frac{\pi k}{2}}{2 \sinh \frac{\pi p k}{2} \cosh \frac{\pi k}{2}},
$$

and $\chi(\theta)$ is related to the SUSY factor of the S matrix

$$
\chi(\theta)=1 /(2 \pi \cosh \theta)
$$

The exact ground state energy depends only on the NLIE-like function via $y_{1}(\theta)$

$$
E(l)=-\frac{i}{2 \pi}\left(\int_{\mathcal{C}_{1}} d \theta \sinh \theta \ln \left(1+y_{1}(\theta)\right)-\int_{\mathcal{C}_{2}} d \theta \sinh \theta \ln \left(1+y_{1}^{-1}(\theta)\right)\right) .
$$

By setting the parameters $\omega$ and $\delta$ appropriately these equations provide access to all three vacua, though not for all possible choices of $(\beta, l)$ for each state, as we shall comment on further below. The appropriate settings are shown in table 5.1.

Typically we can extract a number of results analytically from nonlinear integral equations, the simplest of which is the ultraviolet value of the ground state energy. In the Neveu-Schwarz sector $(\delta=0)$ the scaling function behaves as :

$$
\frac{6 l}{\pi} E(0)=-\left(\frac{3}{2}-\frac{12 \omega^{2}}{r^{2}}\right)
$$




\begin{tabular}{|l|c|c|}
\hline State & $\omega$ & $\delta$ \\
\hline$|\tilde{0}\rangle \otimes|N S\rangle$ & 0 & 0 \\
\hline$|1+\rangle$ & 1 & 0 \\
\hline$|\tilde{0}\rangle \otimes|R\rangle$ & $\frac{1}{2}$ & 1 \\
\hline
\end{tabular}

Table 5.1: Choice of NLIE parameters to obtain each of the three ground states.

while in the Ramond sector $(\delta=1)$ we have

$$
\frac{6 l}{\pi} E(0)=-\left(-\frac{12(\omega-1 / 2)^{2}}{r^{2}}\right)
$$

Note that tuning $\omega$ according to Table 5.1 yields the correct UV behaviour for each ground state:

$$
\frac{6 l}{\pi} E(0)=-\left(c-24 \Delta_{a}\right)
$$

Furthermore the conformal dimension of the perturbing operator exactly matches that of $U_{1}$. In the infrared limit $(l \rightarrow \infty)$ we deduce the scaling functions behaviour to be [5]

$$
E(l) \sim-2 \sqrt{2} \cos \pi \omega \int \frac{d \theta}{2 \pi} \cosh \theta e^{-M l \cosh \theta} .
$$

This result can be given a simple intuitive interpretation. First, let us note that the RSOS structure describing the SUSY degrees of freedom of solitons has three nodes $0,1 / 2$, 1 , which correspond to truncating the quantum group representation theory $\mathcal{U}_{q}(s l(2))$ at $q^{4}=1$ to the tensor product rule

$$
\begin{aligned}
& 0 \otimes a=a \quad, \quad a=0, \frac{1}{2}, 1 \\
& \frac{1}{2} \otimes \frac{1}{2}=0 \oplus 1 \quad, \quad 1 \otimes 1=0 .
\end{aligned}
$$

There is a notion of statistical dimension for these representations $d_{a}(a=0,1 / 2,1)$, which satisfies the ordinary rules under the tensor product

$$
\begin{aligned}
& d_{0} d_{a}=d_{a} \quad, \quad a=0, \frac{1}{2}, 1 \\
& d_{\frac{1}{2}}^{2}=d_{0}+d_{1} \quad, \quad d_{1}^{2}=d_{0},
\end{aligned}
$$

and the solution to these conditions is

$$
d_{0}=d_{1}=1 \quad, \quad d_{\frac{1}{2}}=\sqrt{2} .
$$

The kinks correspond to the $1 / 2$ representation, and the above results mean that they have a statistical dimension $\sqrt{2}$. It might seem strange since it is hard to interpret this as the 


\begin{tabular}{|c|c|c|}
\hline$r$ & $b_{2}$ & $c_{2}^{a} \kappa^{2}$ \\
\hline$\sqrt{15} / 3$ & -0.4305230886667 & -0.4305230886637 \\
\hline$\sqrt{21} / 3$ & -0.7099942272385 & -0.7099942272382 \\
\hline 2 & -0.5922281367467 & -0.5922281367458 \\
\hline 3 & -0.2517882823698 & -0.2517882823567 \\
\hline 3.05 & -0.2422117898616 & -0.2422117898994 \\
\hline
\end{tabular}

Table 5.2: Comparison of PCFT correction with NLIE for the first Neveu-Schwarz ground state

multiplet length. However, a simple calculation shows that the number of $2 n$-kink states (neglecting for the moment the topological charge) with periodic boundary conditions grows as $2^{n}$ (there are no odd-kink states on a circle) so the kinks indeed behave as a multiplet of length $\sqrt{2}$. The reason for this can be found in the nontrivial vacuum adjacency conditions imposed on multi-kink states, which do not allow for all possible sequences to be realized.

Armed with this, it is easy to interpret the result (5.1). For $\omega=0$ it is exactly the leading correction from $2 \sqrt{2}$ particles in finite volume (or equivalently finite temperature) to the free energy. The additional factor 2 comes from the fact that the topological charge introduces a further doublet structure of the kinks. The $\omega$ dependence is easy to understand using the same sort of instanton argument as in the case of the ordinary sine-Gordon model [11]. ${ }^{2}$

A final check can be made by comparing NLIE data with the above perturbed conformal theory predictions. Turning to the first Neveu-Schwarz ground state, we extracted the coefficient $b_{2}$ from NLIE data fitted to a suitably truncated series of the form

$$
\frac{6 l}{\pi} E_{a}(l)=-\left(c-24 \Delta_{a}\right)-\sum_{n=1} b_{2 n} l^{2 n y},
$$

then made a comparison with the coefficient of the leading PCFT correction $c_{2}^{a} \kappa^{2}$. The results displayed in Table 5.2 show excellent agreement. Numerical iteration of the NLIEs in the Ramond sector confirms the Ramond ground state energy is identically zero for all choices of $l$. This provides a nontrivial check of the equations since the NLIE functions $y_{1}(\theta)$ and $y_{2}(\theta)$ are not identically zero.

It turns out to be harder to check the NLIEs for the second Neveu-Schwarz ground state. For small values of $r$ and large values of the cylinder size $l$ the equations work well. This allows a comparison with PCFT, but does not allow one to obtain the value of $c_{2}^{a} k^{2}$ to

\footnotetext{
${ }^{2}$ We remark that a similar explanation can be made for the IR behaviour of the vacuum scaling function predicted by the NLIE, for all the fractional supersymmetric sine-Gordon models, c.f. formula (29) in [5]. For the case $L$ of that paper, the statistical dimension of the kink representation $1 / 2$ results in $2 \cos (\pi /(L+2))$. SSG corresponds to $L=2$, and ordinary sine-Gordon to $L=1$ (i.e. the kinks have dimension 1 apart from the double degeneracy corresponding to their topological charge).
} 


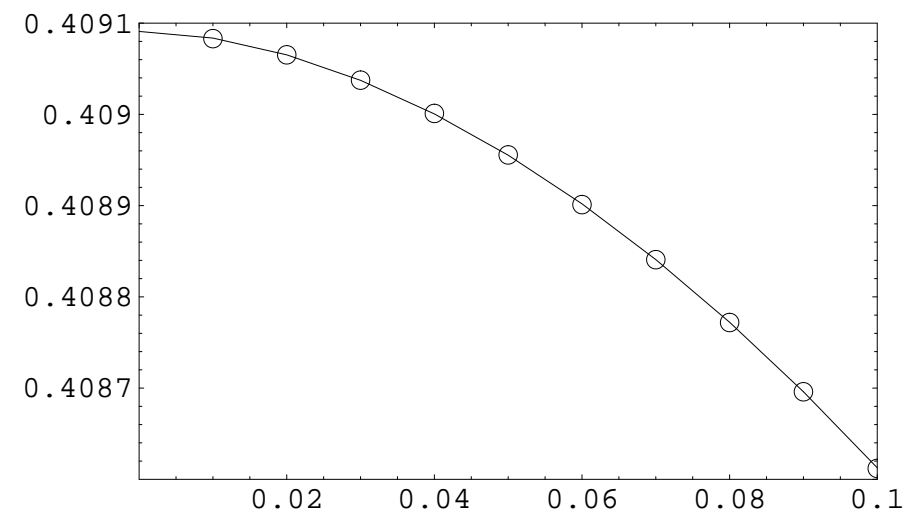

Figure 5.1: Comparing PCFT with NLIE for the second Neveu-Schwarz ground state

as many decimal places as for the lower NS ground state. Instead, in Fig. 5.1 we compare the PCFT result at compactification radius $r^{2}=11$ (equivalently $p=1 / 5$ )

$$
c-24 \Delta_{|1+\rangle}+c_{2}^{|1+\rangle} k^{2} l^{2 y}
$$

using a solid line to the NLIE data $-6 l E_{a}(l) / \pi$ (indicated by the symbol $\circ$ ). The agreement is very good.

So what lies behind the difficulties at some couplings and/or cylinder size? For larger values of $r$ or smaller $l$ the equations fail because one or more zeros of $1+y_{1}(\theta)$ attempt to cross one of the integration contours, resulting in a singularity in $\ln \left(1+y_{1}(\theta)\right.$ ). (Once this has happened a zero of $1+y_{2}(\theta)$ may also attempt to cross a contour.) This behaviour is not unexpected: by sending the cylinder size $l$ into the complex plane the authors of $[28,29]$ were able to analytically continue a set of TBA equations describing the ground state energy of a particular model to a new set, which gave access to the first excited state. Here, we have an additional parameter $\omega$ which we analytically continue from 0 to 1 along the real axis to obtain the second NS ground state from the first. Singularities in the logarithmic term of NLIEs of the type discussed here also arise via a second mechanism, which usually occurs as the (real) cylinder size $l$ is decreased and is not explicitly connected to an excited state. This so-called 'specials' problem was first described in the context of the ordinary sine-Gordon NLIE when the equation was tuned to study the various states of the minimal models perturbed by $\phi_{13}$ [30]. Since we are able to make a favourable comparison of NLIE data and PCFT results for some couplings $r$ we leave the resolution of these problems for future investigation. This will also provide clues as how to modify the NLIEs to address the excited states, as has been done for the sine-Gordon NLIE in [9,31-34]. 


\section{TCSA}

\subsection{TCSA for the supersymmetric sine-Gordon model}

The supersymmetric sine-Gordon model can be viewed as a relevant perturbation of the

$c=\frac{3}{2}$ free conformal field theory, consisting of a free fermion and a free boson. Following the idea of the truncated conformal space approach (TCSA) [35], the Hilbert space is truncated at a given conformal energy $E_{\text {cut }}$, and so the Hamiltonian can be diagonalized numerically to obtain an approximate finite volume spectrum. Since the perturbing operator has scaling dimension larger than $1 / 2$ we are faced with divergences in the TCSA data and hope to obtain relative energy levels only. Furthermore, the Hilbert space contains many more vectors up to a given energy cut than in a theory with a single scalar field only, so only qualitative results are expected.

The matrix elements of the perturbing operator between any two conformal states $|a\rangle$ and $|b\rangle$ with conformal weights $\left(\Delta_{a}, \bar{\Delta}_{a}\right)$ and $\left(\Delta_{b}, \bar{\Delta}_{b}\right)$ can be brought to the following form

$\left\langle a\left|g \int_{0}^{L} d x \chi(\xi, \bar{\xi})\right| b\right\rangle=\frac{g L}{\sqrt{2}}\left(\frac{2 \pi}{L}\right)^{2 \Delta}\left\langle a\left|i \psi(1) \bar{\psi}(1)\left(V_{(1,0)}(1,1)+V_{(-1,0)}(1,1)\right)\right| b\right\rangle \delta_{\Delta_{a}-\bar{\Delta}_{a}, \Delta_{b}-\bar{\Delta}_{b}}$

using a conformal mapping from the cylinder to the plane and translational invariance (the $\delta$ expresses the conservation of momentum/conformal spin). Recall that $\Delta=\frac{1}{2}+\frac{1}{2 r^{2}}$.

We use relations $(4.1,4.3)$ to write the PCFT coupling $g$ in terms of the kink mass $M$ and to convert the Hamiltonian into dimensionless form (energy measured in units of $M)$, parametrized by the dimensionless volume parameter $l=M L$. The Hilbert space can be decomposed into sectors of given conformal spin and given bosonic winding number (topological charge).

Using the mode expansions and $U(1)$ charge conservation, the matrix elements of the perturbing operator can be calculated as the product of separate bosonic and fermionic contributions. In the Ramond sector, matrix elements including the fermion zero mode can be computed using the relation

$$
\left\langle R\left|i b_{0} \bar{b}_{0}\right| R\right\rangle=\frac{1}{2}
$$

Since the interaction is even in the bosonic field, the perturbing operator has the following discrete bosonic $Z_{2}$ symmetry

$$
|n, m\rangle \leftrightarrow|-n,-m\rangle \quad ; \quad a_{n} \leftrightarrow-a_{n} \quad ; \quad \bar{a}_{n} \leftrightarrow-\bar{a}_{n}
$$

The discrete symmetry $(2.4,2.5)$ means that the NS sector has an additional $Z_{2}$ symmetry acting as

$$
|n, m\rangle \leftrightarrow(-1)^{n}|n, m\rangle \quad ; \quad b_{s} \leftrightarrow-b_{s} \quad, \bar{b}_{s} \leftrightarrow \bar{b}_{s}
$$

There is no such symmetry in the Ramond sector as a result of the presence of the fermionic zero mode. 


\begin{tabular}{|c|c|c|c|c|c|}
\hline$++\quad$ (口) & $-+\quad(\circ)$ & +- & -- & $(\triangle)$ & $\begin{array}{ll}- & (+) \\
\end{array}$ \\
\hline$\overline{|c| 0\rangle}$ & $|1+\rangle$ & & & $\overline{\mid \overline{|0\rangle_{R}}}$ & \\
\hline & & $|2-\rangle$ & $|1-\rangle$ & & $|1-\rangle_{R}$ \\
\hline$|2+\rangle$ & $|3+\rangle$ & & & $|1+\rangle_{\mathrm{B}}$ & \\
\hline
\end{tabular}

Table 6.1: UV classification of the lowest lying states in the various NS and R sectors for $r>3$. For $r<3$ the order of the states is somewhat different $(|3+\rangle$ is the third state in its sector), but they can still be identified unambiguously.

The Hilbert space (see Section 4.1) can be further decomposed with respect to these symmetries. In table 6.1 we list the lowest energy states of each sector for zero spin and zero topological charge. The first sign refers to the NS parity, while the second to the bosonic one. In parenthesis we indicate the symbol we used in the TCSA data for the various sectors and $|N \pm\rangle_{\mathrm{R}}=\frac{1}{\sqrt{2}}\left(V_{(N, 0)}(0,0)|0\rangle_{\mathrm{R}} \pm V_{(-N, 0)}(0,0)|0\rangle_{\mathrm{R}}\right)$, where $|0\rangle_{\mathrm{R}}=|\tilde{0}\rangle \otimes|R\rangle$.

\subsection{Checking TCSA against PCFT}

The aim of this investigation is to confirm the TCSA program by comparing the small volume (UV) data for the low lying energy levels to the predictions of conformal perturbation theory (PCFT). Since the TCSA data are given in terms of the (IR) kink mass while the PCFT predictions in terms of the (UV) parameter $\mu$, to make the formal comparison one has to use the massgap relation. Nevertheless as the TCSA program is written using the same massgap relation the success of the comparison says nothing about the correctness of this relation but may confirm the TCSA program.

Since the scaling dimension of the perturbing operator is larger than $1 / 2$ both the conformal perturbation theory and TCSA are plagued by divergences. As discussed in section 3.3, in PCFT these divergences are regularized by analytical continuation. The introduction of a finite $E_{\text {cut }}$ in TCSA also regularizes these divergences albeit in a different way. Therefore the best one can do to compare TCSA and the PCFT predictions is to consider the differences between the various energy levels rather than the levels themselves:

$$
\frac{6 l}{\pi}\left(E_{b}(l)-E_{a}(l)\right)-24\left(\Delta_{b}-\Delta_{a}\right)=\Delta c^{b a} \kappa^{2} l^{2 y}+o\left(l^{4 y}\right), \quad \Delta c^{b a}=c_{2}^{a}-c_{2}^{b} .
$$

This has two advantages: on the one hand the TCSA energy differences depend less sensitively on $E_{\text {cut }}$ than the individual levels, and on the other the integrals defining $\Delta c^{b a}$ are convergent thus they may be computed as the appropriate differences of the data in the table 4.1 .

Using the identifications between the low lying TCSA lines and the $|N \pm\rangle$ states in table 6.1 we compare the TCSA energy differences and the PCFT predictions in Fig. 6.1. In diagram (a), the three continuous lines depict the PCFT predictions with $\Delta c^{|1-\rangle|0\rangle} \Delta c^{|1+\rangle|0\rangle}$ and $\Delta c^{|2+\rangle|0\rangle}$ respectively, while - in accordance with table 6.1 - the symbols $*, \circ, \square$ and 
$x$ denote the TCSA data for

$$
\begin{aligned}
& \left(E_{1}^{--}(l)-E_{1}^{++}(l)\right) \frac{6 l}{\pi}-\frac{12}{r^{2}} \\
& \left(E_{1}^{-+}(l)-E_{1}^{++}(l)\right) \frac{6 l}{\pi}-\frac{12}{r^{2}} \\
& \left(E_{2}^{++}(l)-E_{1}^{++}(l)\right) \frac{6 l}{\pi}-\frac{48}{r^{2}} \quad \text { and } \\
& \left(E_{1}^{+-}(l)-E_{1}^{++}(l)\right) \frac{6 l}{\pi}-\frac{48}{r^{2}}
\end{aligned}
$$

respectively. Note that the data is consistent with the $|2 \pm\rangle$ states being degenerate in the leading order of PCFT.

In diagram (b), the first three lines and the first four sets of TCSA data are just like on (a), while the fourth continuous line depicts the leading PCFT prediction for $\Delta c^{|3+\rangle|1+\rangle}$ and the second line of points marked by $\circ$ are the TCSA data

$$
\left(E_{3}^{-+}(l)-E_{1}^{-+}(l)\right) \frac{6 l}{\pi}-\frac{96}{r^{2}} .
$$

The agreement between the data and the leading predictions is excellent, though the deviations between them indicate that in some cases the higher order PCFT corrections are not negligible even in this $l$ region.

\subsection{IR spectrum from TCSA}

Since the scaling dimension of the perturbing operator is larger than $1 / 2$ we have divergences in the TCSA data. This can be seen by increasing the cutoff: the energy levels become more and more negative. The energy differences, however, converge but unfortunately very slowly. The first two lines in the $(++),(-+) \mathrm{NS}$ and in the $(+) \mathrm{R}$ sectors, and the first (lowest) lines in the (+-), (--) NS and (-) R sectors are shown in Fig. 6.2, where we plot these lines relative to the ground state of the $(++)$ NS sector.

The various points are marked according to table 6.1. The first row in the table corresponds to the UV limit of the three vacua as shown on the data. The next row, (the next three curves) corresponds to the first breathers excited above each vacua, while the last row, (the last three curves) are related to the second breathers. The bosonic breathers are identified on the basis of their behaviour under the discrete symmetries. The compactification radius is $r=3.05$ and the energy cut is 14 , which corresponds to approximately 13000 states in the Ramond sector and 9000 states in the NS sectors, respectively. Clearly the curves are in a qualitative agreement with the spectrum, but the particular mass values are wrong. Indeed it can be seen that the TCSA converges very slowly to the right values: by changing the truncation level from 8 to 14 in steps of 2 , the energy differences change almost the same amount in each step. However, the qualitative features of the spectrum (i.e. the degeneracy and gap patterns) already appear for very small number of states and are stable against increasing the truncation level. 


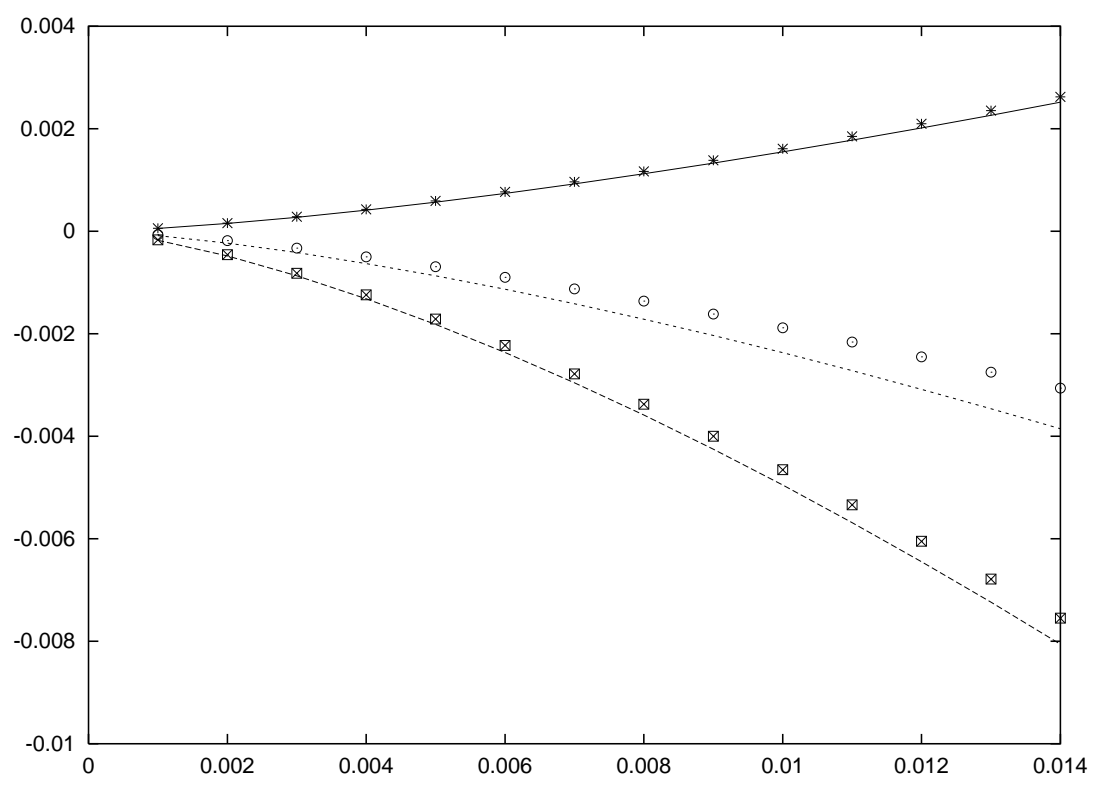

(a) $\mathrm{r}=1.9$

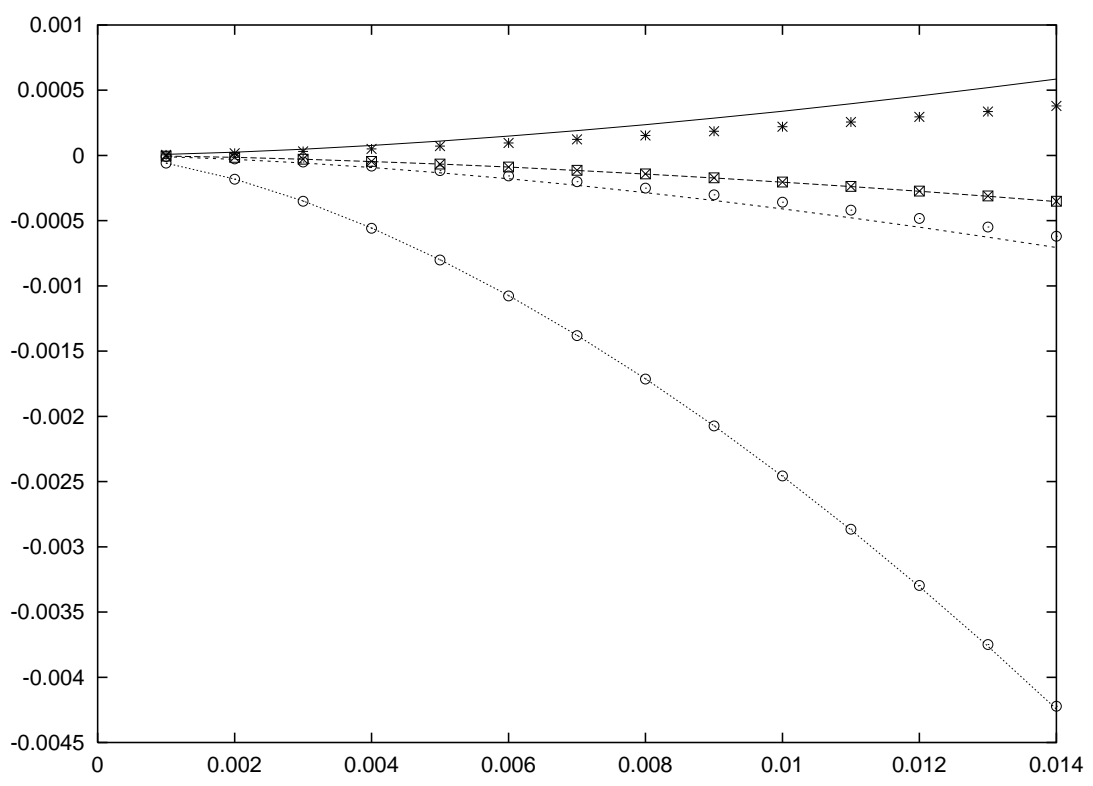

(b) $\mathrm{r}=2.3$

Figure 6.1: Comparison of TCSA and PCFT energies relative to the NS $(++)$ vacuum 


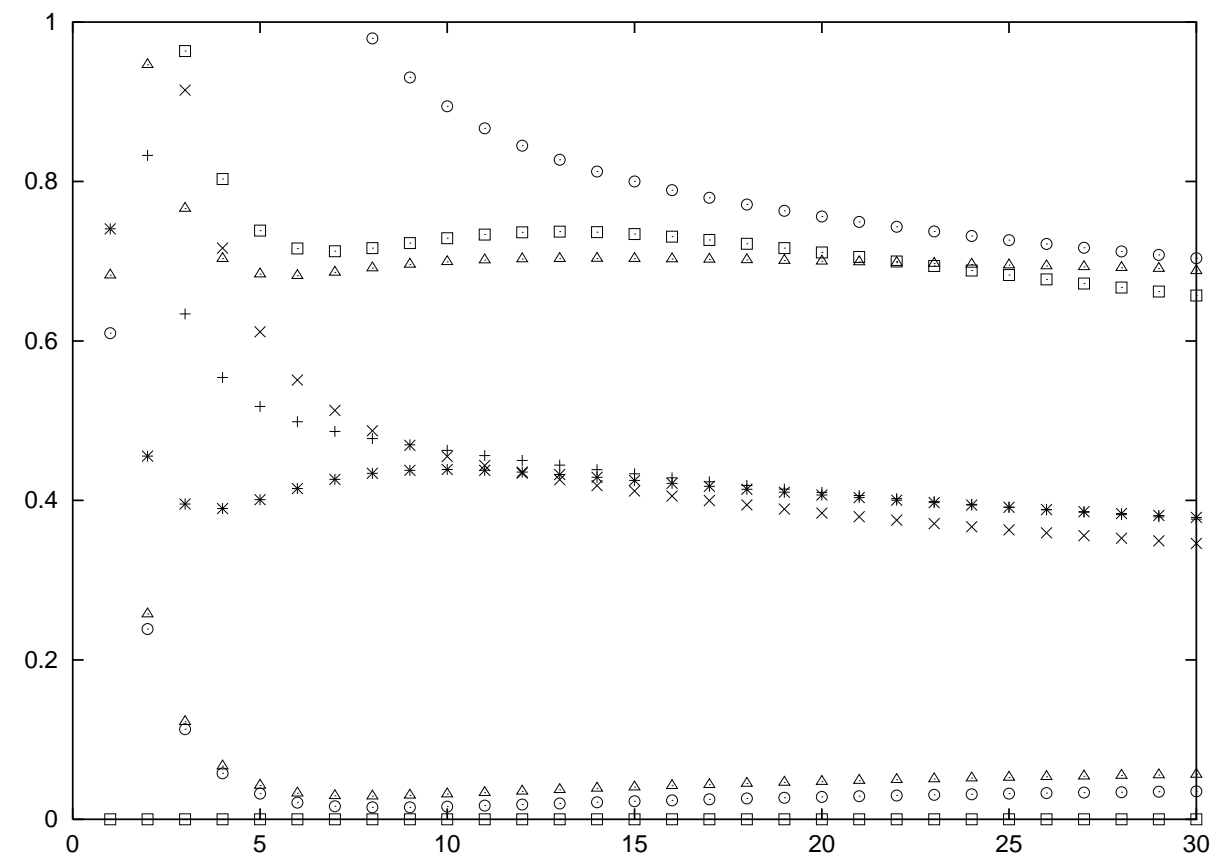

Figure 6.2: Lowest lying states in the TCSA spectrum

Even though the mass values converge very slowly, the case for the mass ratios is much better. Calculating particular mass ratios from the data, the predictions of S matrix theory (2.9) can be reproduced with a few percent accuracy, and are much more stable against changing the cut.

Furthermore, the TCSA data are also qualitatively consistent with the dependence of the spectrum on the coupling, i.e. by changing the compactification radius $r$ the number and relative position of breather line triplets follows the predicted spectrum.

\section{Conclusions}

In this work we investigated supersymmetric sine-Gordon theory (SSG), especially the structure of the vacua and the kinks connecting them. Using an argument due to Zamolodchikov we clarified the vacuum and kink structure and showed that it corresponds exactly to the exact $\mathrm{S}$ matrix conjectured in [1]. Then we formulated the theory in the perturbed conformal field theory (PCFT) framework, which shows a striking difference to the usual Lagrangian description, namely the omission of a purely scalar potential term. The first evidence we gave for the correctness of this description came from the construction of a spin-3 conserved charge, which is necessary for integrability. Considering the classical limit, we showed that the expression for this charge obtained in the PCFT framework reduces to the result known from the classical Lagrangian approach, evidence that the two approaches 
indeed describe the same model.

Using PCFT, we obtained the leading behaviour of some energy levels in finite volume, for the limit of small volume ( the ultraviolet regime). In particular, we concluded that the energy of the Ramond ground state vanishes in this limit, which is consistent with the fact that it is expected to vanish exactly due to unbroken SUSY in finite volume in this sector.

The PCFT results were then compared to results obtained from an NLIE equation for the lowest lying Neveu-Schwarz state, which is the true ground state in finite volume. This comparison involves the mass gap relation between the PCFT coupling (an UV parameter) and the soliton mass (an IR quantity) conjectured earlier in [17]. The comparison showed excellent agreement for several different values of the dimensionless coupling parameter $\beta$ (equivalently $r$ ) of the model, which is a strong evidence for the correctness of the mass gap formula on one hand, and for the consistency between the PCFT and NLIE approach on the other.

We have also examined the large volume (infrared) limit of the NLIE and found a perfect agreement with considerations based on statistics of kinks and instanton calculus in finite volume.

The NLIE proposed in [5] was formulated for the true ground state of SSG, which is the lowest lying state in the Neveu-Schwarz sector. Matching conformal dimensions computed from the NLIE with those predicted from conformal field theory, we proposed equations describing the other two vacua, one in the Ramond and the other in the Neveu-Schwarz sector. Numerical iteration of the NLIE for the Ramond ground state gave a vanishing energy for all volumes, as expected, providing a very strong check since the solution of the equation itself is still nontrivial in this case. For the second Neveu-Schwarz vacuum, convergence properties of the iteration are not as good as for the other two cases, but the UV behaviour matches the PCFT prediction nicely, giving another confirmation of the consistency between PCFT, NLIE and the massgap formula.

Finally, we performed an analysis of the finite volume problem using truncated conformal space approach (TCSA). We checked the validity of the approach in the UV regime by a comparison with PCFT. While the numerical convergence of the TCSA is not very good (partially due to UV divergences, but also to a rapidly growing number of states as the energy cut is raised), it does confirm the qualitative picture of the spectrum, both the degeneracies and the behaviour of the breather levels are in accordance with the picture presented before.

There are many open issues. It would be very interesting to give a complete characterization of the SSG finite volume spectrum from the NLIE, like it has been done in the non-SUSY case $[9,25,26,30-34]$. It is not at all obvious how to achieve that, however, mainly because the NLIE for the SSG theory is of a mixed type - part of the system resembles the Destri-de Vega equation, while the other part looks like a thermodynamic Bethe Ansatz equation. It is likely that a reformulation of the NLIE is necessary.

Another interesting problem would be to extend the analysis of the spectrum to the boundary case, along the lines of non-SUSY sine-Gordon theory [36]. This was part of the motivation to introduce TCSA for SSG, however, the slow convergence of TCSA prevents 
us from obtaining reliable quantitative results, which would be necessary to check the bootstrap spectrum proposed in [37]. It remains to be seen whether this can be overcome in some way. Extending the NLIE to the boundary situation could be another way to obtain interesting results and check the bootstrap predictions.

\section{Acknowledgments}

The authors would like to thank Gerard Watts for illuminating discussions. We are also grateful to Marco Moriconi for sharing with us his unpublished TBA results on Fendley's scattering theory. TCD thanks the UK EPSRC for a Research Fellowship. This work was partially supported by the EC network "EUCLID", contract number HPRN-CT-200200325, and Hungarian research funds FKFP 0043/2001, OTKA D42209, T037674, T034299 and T043582. GT was also supported by a Széchenyi István Fellowship, and ZB by a Bolyai János Research Fellowship.

\section{References}

[1] C. Ahn: Nucl. Phys. B354 (1991) 57-84.

[2] H. Nastase, M. A. Stephanov, P. van Nieuwenhuizen and A. Rebhan: Nucl. Phys. B542 (1999) 471-514, hep-th/9802074.

N. Graham and R. L. Jaffe, Nucl. Phys. B544 (1999) 432-447, hep-th/9808140.

M. A. Shifman, A. I. Vainshtein and M. B. Voloshin, Phys. Rev. D59 (1999) 045016, hep-th/9810068.

A. Rebhan, P. van Nieuwenhuizen and R. Wimmer, Nucl. Phys. B648 (2003) 174-188, hep-th/0207051.

[3] P. Fendley: Phys. Lett. B250 (1990) 96-101.

[4] A.B. Zamolodchikov: Fractional-spin integrals of motion in perturbed conformal field theory, in Fields, Strings and Quantum Gravity, eds. H. Guo, Z. Qiu and H. Tye (Gordon and Breach, 1984).

[5] C. Dunning: J. Phys. A36 (2003) 5463-5476, hep-th/0210225.

[6] S. Ferrara, L. Girardello and S. Sciuto: Phys. Lett. B76 (1978) 303-306.

[7] K. Schoutens: Nucl. Phys. B344 (1990) 665-695.

[8] T.R. Klassen and E. Melzer: Int. J. Mod. Phys. A8 (1993) 4131-4174, hep-th/9206114.

[9] G. Feverati, F. Ravanini and G. Takács: Phys. Lett. B444 (1998) 442-450, hepth/9807160.

[10] A.B. Zamolodchikov: Phys. Lett. B335 (1994) 436-443. 
[11] Z. Bajnok, L. Palla, G. Takács and F. Wágner: Nucl. Phys. B587 (2000) 585-618, hep-th/0004181.

[12] T. Hollowood and A. Mavrikis: Nucl. Phys. B484 (1997) 631, hep-th/9606116.

[13] A.B. Zamolodchikov: Int. J. Mod. Phys. A3 (1988) 4235; Adv. Stud. Pure Math. 19 (1989) 614.

[14] H.C. Liao and P. Mansfield: Nucl. Phys. B344 (1990) 696.

[15] P. Mathieu: Nucl. Phys. B336 (1990) 338-348;

P. Mathieu and G.M.T. Watts: Nucl. Phys. B510 (1998) 577-607, hep-th/9707050.

[16] T. Inami, S. Odake and Y.Z. Zhang: Phys. Lett. B359 (1995) 118-124, hepth/9506157;

R.I. Nepomechie: Phys. Lett. B509 (2001) 183-188, hep-th/0103029.

[17] P. Baseilhac and V.A. Fateev: Nucl. Phys. B532 (1998) 567-587, hep-th/9906010.

[18] T.R. Klassen and E. Melzer: Nucl. Phys. B350 (1991) 635-689.

[19] C.N. Yang and C.P. Yang: J. Math. Phys. 10 (1969) 1115-1122.

[20] Al.B. Zamolodchikov: Nucl. Phys. B342 (1990) 695-720.

[21] P. Fendley and K. Intriligator: Nucl. Phys. B372 (1992) 533-558, hep-th/9111014.

[22] F. Ravanini, R. Tateo and A. Valleriani: Int. J. Mod. Phys. A8 (1993) 1707-1727, hep-th/9207040.

[23] R. Tateo: Int. J. Mod. Phys. A10 (1995) 1357-1376, hep-th/9405197.

[24] R. Tateo: Phys. Lett. B355 (1995) 157-164, hep-th/9505022.

[25] C. Destri and H.J. de Vega: Phys. Rev. Lett. 69 (1992) 2313-2317; Nucl. Phys. B438 (1995) 413-454, hep-th/9407117.

[26] A. Klümper and P.A. Pearce: J. Stat. Phys. 64 (1991) 13-76.

[27] A. Klümper, M.T. Batchelor and P.A. Pearce: J. Phys. A24 (1991) 3111-3133.

[28] P. Dorey and R. Tateo: Nucl. Phys. B482 (1996) 639-659, hep-th/9607167.

[29] P. Dorey and R. Tateo: Nucl. Phys. B515 (1998) 575-623, hep-th/9706140.

[30] G. Feverati, F. Ravanini and G. Takács: Nucl. Phys. B570 (2000) 615-643, hepth $/ 9909031$.

[31] D. Fioravanti, A. Mariottini, E. Quattrini and F. Ravanini: Phys. Rev. Lett. B390 (1997) 243-251, hep-th/9608091. 
[32] C. Destri and H.J. de Vega: Nucl. Phys. B504(1997) 621-664, hep-th/9701107.

[33] G. Feverati, F. Ravanini and G. Takács: Phys. Lett. B430 (1998) 264-273, hepth/9803104.

[34] G. Feverati, F. Ravanini and G. Takács: Nucl. Phys. B540 (1999) 543-586, hepth/9805117.

[35] V. P. Yurov and A. B. Zamolodchikov: Int. J. Mod. Phys. A5 (1990) 3221;

V. P. Yurov and A. B. Zamolodchikov: Int. J. Mod. Phys. A6 (1991) 4557.

[36] Z. Bajnok, L. Palla and G. Takács, Nucl. Phys. B614 (2001) 405, hep-th/0106069;

Z. Bajnok, L. Palla and G. Takács, Nucl. Phys. B622 (2002) 565, hep-th/0108157.

[37] Z. Bajnok, L. Palla and G. Takács, Nucl. Phys. B644 (2002) 509, hep-th/0207099. 